\title{
GNSS AND PHOTOGRAMMETRY BY THE SAME TOOL: A FIRST EVALUATION OF THE LEICA GS18I RECEIVER
}

\author{
V. Casella ${ }^{1 *}$, M. Franzini ${ }^{1}$, A. M. Manzino ${ }^{2}$ \\ ${ }^{1}$ Dept. of Civil Engineering and Architecture, University of Pavia, Pavia, Italy - (vittorio.casella, marica.franzini)@ unipv.it \\ ${ }^{2}$ Dept. of Environment, Land and Infrastructure Engineering, Polytechnic of Turin - Torino, Italy - \\ ambrogio.manzino@formerfaculty.polito.it
}

Commission II, WG II/10

KEY WORDS: Integration, GNSS, Photogrammetry, Direct Sensor Orientation.

\begin{abstract}
Leica Geosystems recently introduced a multi-constellation GNSS sensor named GS18i. It is capable to perform tilt compensation and has an integrated photogrammetric camera, allowing the users to measure inaccessible features: this is called visual positioning. The Laboratory of Geomatics, at the University of Pavia - Italy, performed a first evaluation of the rover. Five accessible points were measured repeatedly with the pole having different tilt angles; measurements' total number was 2077. After moderate blunder detection, RMSE values are 12, 10 and $18 \mathrm{~mm}$, for the East, North and height components. Measurement quality is substantially independent from the pole's tilt angle. Moreover, ten points belonging to a building's façade were repeatedly measured by photogrammetry, through the integrated camera, from distances in the range between 4 and 12 meters. In total, 1436 measurements were acquired. After blunder detection, RMSE values are 45, 25 and $66 \mathrm{~mm}$, for the $x, y$ and $z$ components of a local cartesian system. Measurement quality mildly depend on the object-camera distance. Despite a good overall accuracy, results show some surprising aspects: the high ratio between the planimetric component $x$ and $y$, the counterintuitive behaviour of the $y$ dispersion, which decreases when the distance increases. While the present paper aims at simply being a first evaluation of the rover, next activities will deal with rigorous and controlled photogrammetric processing of the images and will also include simulations, in order to ascertain the role played by the various error sources involved.
\end{abstract}

\section{INTRODUCTION}

Leica Geosystems recently introduced a multi-constellation GNSS sensor named GS18i ("Leica GS18I," 2020). Together with the usual functionalities of a modern GNSS receiver, it is capable to perform tilt compensation, thanks to the onboard IMU (Inertial Measuring Unit). Moreover, it has an integrated photogrammetric camera (Figure 1), allowing the users measuring inaccessible features. It can be said it implements the integration of GNSS and photogrammetry. As a confirmation, the company speaks about visual positioning.

Very interestingly, the controller's onboard software can orient the images in the way called, in the photogrammetric jargon, direct sensor orientation. To do so, it integrates GNSS measurements, those coming from the accelerometers and information obtained by image matching.

The Laboratory of Geomatics, at the University of Pavia, Italy, had the equipment on loan from Leica Geosystems Italy, for a first evaluation and then acquired it. The Laboratory conducted a rather extensive validation, which is partly illustrated in the present paper.

\section{RELATED LITERATURE}

Integrated survey was one of the most important trend topics in the last two decades. Combinations of GNSS receivers, INS systems, LiDAR devices and cameras were developed for supporting MMV (Mobile Mapping Vehicles), UAV (Unmanned Aerial Vehicles) or autonomous driving, creating new opportunities of surveying; computer vision and artificial intelligence were further improved the quality of the results achievable by these systems. Leica GS18I receiver sets perfectly in this trend by combining a GNSS/INS system with a camera inside a unique device.
Preliminary analysis on the integrated use of a GNSS receiver and multi-camera system were proposed in (Baiocchi et al., 2018; Cera and Campi, 2017) in what was called "imaging rover". They used this approach in an extensive series of tests having different morphological and geometric characteristics (archaeology, cultural heritage, geology, etc.) finding good results comparable to those obtained by traditional techniques but with cost effective reduction in terms of logistics and time.

The photogrammetric use of acquired images requires an accurate estimation of the six external parameters: the 3D positions and the attitude of the camera at each shutter click. In the Leica GS18I this is done by combination of the GNSS and INS data, respectively. As the quality of $3 \mathrm{D}$ position is quite wellknown information thanks to the huge experience reached in RTK positioning (El-Mowafy, 2000; Feng and Wang, 2008; Luo et al., 2020), the analysis of attitude still represents a possible research topic.

Receivers usually measure tilt (connected with attitude angles) by means of accelerometers, to determine the inclination, and electronic compass, to establish the direction. Nevertheless, this solution presents some issues: magnetometer inside the compass is influenced by the inclination, an on-site calibration is required and the measurements can be influenced by local magnetic disturbances (Luo et al., 2018). To avoid the drawbacks mentioned above, the tilt compensation solution of the Leica GS18I utilizes precise IMU measurements from industrial-grade micro-electro-mechanical sensors; tests presented in (Luo et al., 2020) shows as the proposed IMU-based tilt compensation is applicable at large tilt angles of more than 30 degrees, where a 3D positioning accuracy of $2 \mathrm{~cm}$ is still achievable.

In Leica GS18I global position and attitude information are then combined with images to measure points in the so called "visual positioning" (Schaufler, 2020). As its release date was in the middle of 2020, there is little literature available for its performance. One of them is (Schaufler et al., 2020) in which the authors present the operating principle and test the receiver under 
different configuration, camera-to-object distances and trajectories varying in length and geometry. Measurement's assessments show high-precision results where 2D and 1D RMS errors are $2.9 \mathrm{~cm}$ and $2.5 \mathrm{~cm}$, respectively.

\section{AIM AND ORGANIZATION OF THE PAPER}

The purpose of the note is to validate precision and accuracy of the photogrammetric measurements performed by the GS18i. As a baseline, results are shown for pure GNSS measurements of accessible benchmarks. In summary, the present paper validates two kinds of measurements:

- $\quad$ Scenario 1 - the GNSS-NRTK measurements of accessible benchmarks, which were performed with the pole kept tilted and the compensation on; as already mentioned, such measurements are not the main focus, in the present paper, but are analysed as a baseline;

- Scenario 2 - photogrammetric measurements of inaccessible points, which are located on a façade of a building. They are obtained from the images acquired by GS18i and from the exterior orientation parameters automatically determined by the receiver.

The paper is organized as follows. Section 4 describes the sensor studied and its operating principle. Sections 5 and 6 describe the test site used and the way the reference coordinates were determined. Section 7 illustrates the two scenarios considered in this paper: measurement of accessible points with the rover mounted on a pole, and with the pole kept tilted; measurement of inaccessible points located on a façade by means of the camera. Sections 8 and 9 present results for the two consider scenarios. Finally, Section 10 discusses the main findings and give some hints on the next planned activities.

\section{BASICS ON THE OPERATING PRINCIPLE OF LEICA GS18I}

The Leica GS18i sensor is a modern, multi-constellation GNSS receiver, capable of acquiring signals from all the systems which are available in Europe, namely GPS, Glonass, Galileo and Beidou.

It usually is operated mounted on top of a pole, though it is still possible to place it on tripods, by suitable adapters. It can perform tilt compensation: even if the pole is not vertical, when a point is measured, the system if able to compensate for the related deviation. Such feature is based on the use of a IMU unit (accelerometers and gyroscopes, plus SW procedures for the integration of instantaneous measurements), rather than inclinometers, which is the most diffused solution for similar instrumentation.

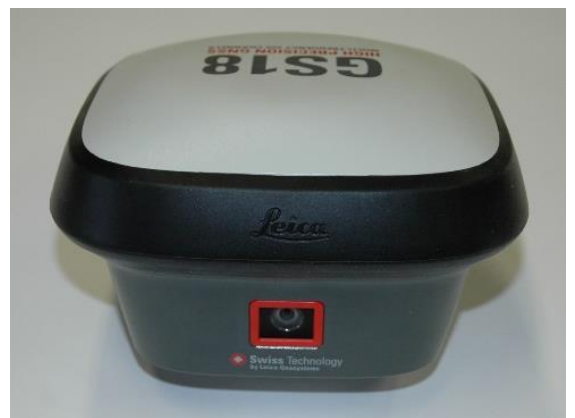

Figure 1. The Leica GS18i GNSS receiver with the camera, in the foreground
The adoption of an IMU, instead of inclinometers, has several consequences. First, quality of tilt compensation is claimed to be better. Furthermore, measurement is sort of dynamic process and tilt compensation is obtained by Kalman filtering; indeed, the pole must be moved to the desired arrangement and the measurement must be performed quite soon, before significant drifts arise; therefore, measurement duration is not set by the user, but determined by the management software, in order to maximize quality. Finally, data coming from the IMU can be used, in conjunction with GNSS observations, to dynamically estimated instantaneous position and orientation of the sensor. This is used when the photogrammetric mode is operated, as illustrated in the next paragraph.

The Leica GS18i sensor is equipped with an integrated ArduCam AR0134 camera ("Arducam AR0134," 2020), which is visible in Figure 1. It is a Bayer-pattern, global shutter, RGB camera having a 1.2 MP image resolution (1280 x 960). Pixel pitch is $3.75 \mu \mathrm{m}$ and focal length is $3.1 \mathrm{~mm}$ for the lens equipping the GS18i; however, the camera manufacturer offers several other options for the lenses. Resolution on the object is $12 \mathrm{~mm}$ at a 10 $\mathrm{m}$ distance. The camera is capable to acquire several tenths of frames per second but, when it is coupled with the GNSS antenna, it is used at the $2 \mathrm{~Hz}$ framing rate. The camera is calibrated so that a full photogrammetric use can be performed. The EXIF file reports fundamental parameters such as calibrated focal length and position of the camera's principal point; concerning lens distortion, we argue that images are undistorted by the controller during the processing happening just after the acquisition; when the images are downloaded into a computer, as we did, they are declared undistorted.

The working principle is the following. When the user is in front of some feature needing visual positioning, such as the façade of a building or a trench in a road, he starts the suitable procedure and then walks in front of the feature, taking care that the camera frames what must be measured. The system automatically acquires an image sequence; by means of GNSS and IMU observations, images' exterior orientation parameters (EOPs) are directly determined. When the user stops the acquisition, the system needs some time to store the data and to refine EOPs by means of tie points, which are automatically extracted, and bundle adjustment. At ("Visual Positioning and Leica GS18 I," 2020), several examples are shown, of how the antenna can be used to acquire buildings, roads, or trenches.

After image storage and pre-processing, it is possible to perform photogrammetric measurements directly on the field, by means of the controller: the user can click on a feature that is visible on one image of the sequence, and the system will automatically search for it in other images and output the coordinates. Nevertheless, it is possible to perform the measurements successively, in the office.

The system typically uses four or five images to perform measurements, even if we could see examples with less or more; indeed, the driving criterion seems to be: all the images where the selected feature can be located with good quality. Also, the system seems quite effective in performing outlier rejection and in discarding wrongly matched features. Indeed, we did not perform a systematic study of this aspect, but the observation of several examples led us to the mentioned conclusion.

The user can anyhow correct homologous point selection and enlarge the set of images used to perform a certain measurement. $\mathrm{He}$ can even fully manually identify features in the various images.

As an example, the image sequences acquired for our test were typically constituted by 30-35 frames, having a storage size of 9.5 $\mathrm{MB}$ and needing around 60 seconds to be processed, after the end of acquisition. 


\section{TEST SITE}

A test site was set up at the Engineering campus of the University of Pavia. It is composed of points belonging to three categories:

- five accessible (which a tripod can be set over) topographic markers, which are shown in Figure 2 in red and are named $100,101,102,103,104$;

- four conners belonging to the reception building; they are shown in Figure 2 again, with the names 201, 202, 203, 204; they won't be considered in this paper, any longer;

- ten points belonging to the North-East façade of the same building; they are displayed in Figure 3 and have names 401-410.

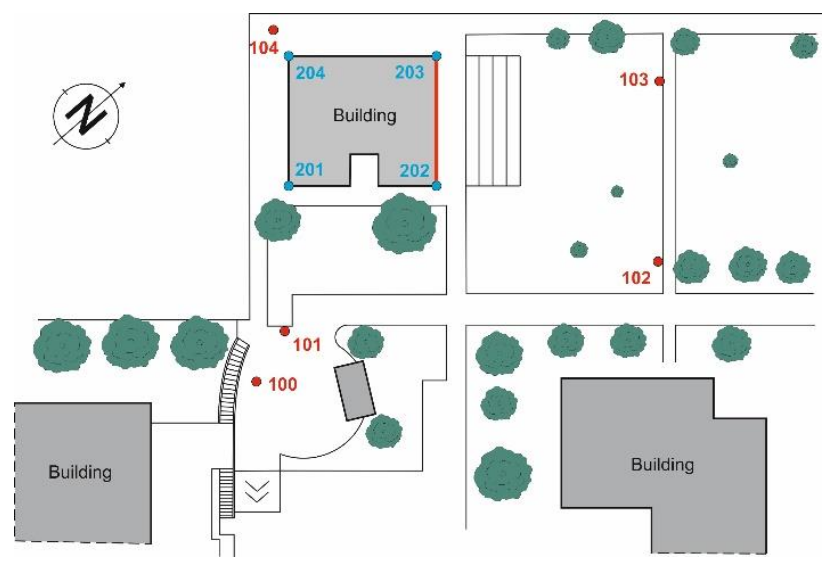

Figure 2. An overview of the test site: the five accessible topographic points are shown in red, whereas the four building corners are highlighted in blue. The façade where are the check points is highlighted in red. Façade points are not visible here, but are shown in Figure 3

As Figure 2 highlights, several points are partially or almost completely covered by trees; moreover, point 100 is close to quite a high wall, which certainly generates a high level of multi-path. All in all, the selected test site is not ideal for receiving GNSS signal but is representative of what a surveyor can meet in daily activities.



Figure 3. The ten points belonging to building's façade.

Points on the facade were surveyed with a redundant topographic network; measurements were processed by least squares adjustment and so-estimated standard deviation values range between 5 and $7 \mathrm{~mm}$.

\section{REFERENCE COORDINATES}

Reference coordinates were determined of the benchmarks, in a very reliable and precise way. The five accessible points $100-$ 104 were surveyed with a redundant, static GNSS survey. The local network was connected to the Leica Smartnet ${ }^{\circledR}$ network of CORS (Continuously Operating Refence Stations), supporting all the available GNSS constellations. After the adjustment, coordinates of the benchmarks were available with an uncertainty (standard deviation) around $1.2 \mathrm{~mm}$ in East and North and 2.3 $\mathrm{mm}$ in height. More details will be given in another, more detailed paper, to be published soon.

\section{REPEATED MEASUREMENT OF BENCHMARKS}

For Scenario 1, three surveyors repeatedly visited points 100 104. The user visited all the five points in sequence, without switching the rover off. At each point, he performed 10 measurements, by leaning the pole in different directions and by different angles. At the end of the round, the antenna was disconnected from the network, so that a new initialization was performed. Number of repeated measurements is reported by Table 1, for each surveyor and each benchmark. Total number is 2077.

\begin{tabular}{|c|c|c|c|c|c|}
\hline $\begin{array}{c}\text { Surveyors/ } \\
\text { points }\end{array}$ & $\mathbf{1 0 0}$ & $\mathbf{1 0 1}$ & $\mathbf{1 0 2}$ & $\mathbf{1 0 3}$ & $\mathbf{1 0 4}$ \\
\hline $\mathbf{A}$ & 151 & 144 & 146 & 147 & 145 \\
\hline $\mathbf{B}$ & 138 & 138 & 138 & 141 & 140 \\
\hline $\mathbf{C}$ & 128 & 129 & 130 & 131 & 131 \\
\hline
\end{tabular}

Table 1. Consistency of measurements acquired in Scenario 1. Report is grouped with respect to the benchmark (100-104) and the surveyor, here named A, B and C

For Scenario 2, repeated photogrammetric measurements of 10 benchmarks were performed. They belong to the façade marked in red in Figure 2 and are shown in Figure 3. The operators walked along the façade at different distances, ranging from 4 to 12 meters from the building. Line followed by operators are shown in Figure 2, just in front of the façade marked in red.

The three surveyors were asked to acquire the façade 10 times for each of the five planned distance steps. They then measured the benchmarks, in the office. In total, 1436 points measurements were performed; each surveyor measured between 445 and 496 points; each benchmark was measured between 138 and 145 times; the various acquisition distances have numerosity in the range between 283 and 290 .

All measurements were performed in the NRTK mode, by connecting the antenna to the Leica Smartnet network. More precisely, the iMAX mode was selected, being a custom Leica mode, which is similar to VRS (Takac and Zelzer, 2008).

Photogrammetric measurements were performed in the office with the Leica Infinity program. The user selects a sequence and then can see miniatures of the acquired images, as illustrated by Figure 4 . He can choose sort of a master image and click on one point to be measured. The program will match the selected template in the neighbouring images and will autonomously decide whether to keep or not an observation, whether to include or not an image; the user is anyway enabled to correct or discard observations and to include new images. By the way, Figure 4 shows the plastic strips we used to guarantee the operator stayed at the planned distance. 


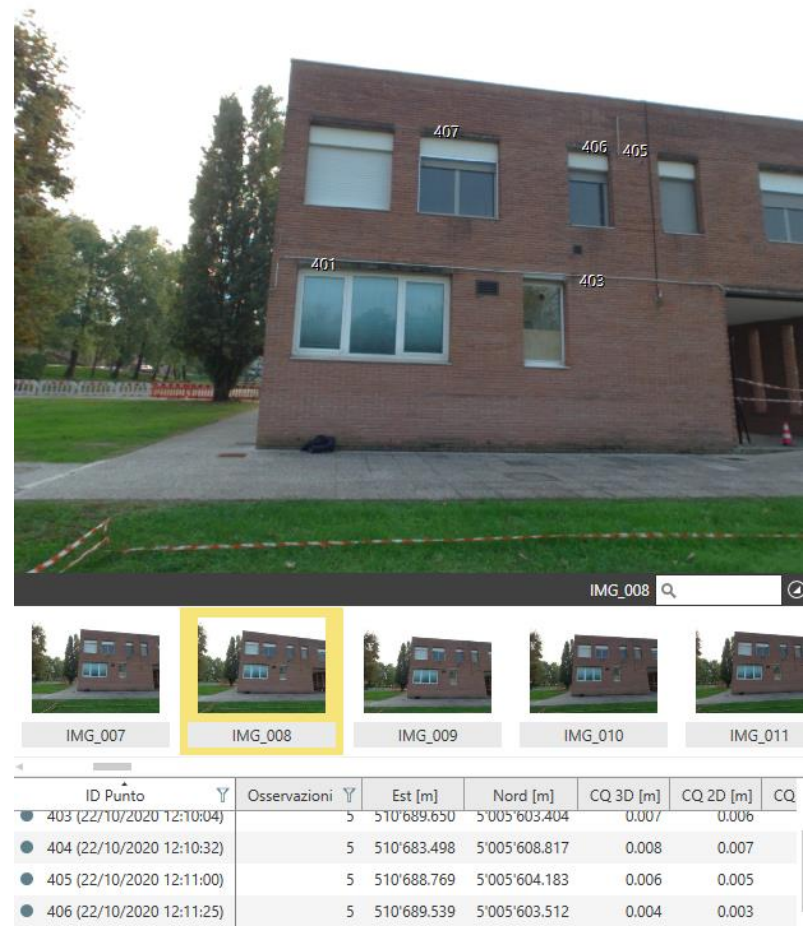

Figure 4. Detail of the interface of the Leica Infinity software by which point measurements were performed in the office.

\section{BASELINE ACCURACY OF TRADITIONAL GNSS MEASUREMENT OF THE ACCESSIBLE BENCHMARKS}

Though this is not the main focus of present paper, results for Scenario 1 are shown, as they constitute a valuable baseline for further considerations. Indeed Scenario 1 allowed us to assess the accuracy, which is attainable in the considered area, with the described instrumentation and by using corrections coming from the mentioned GNSS network. The word traditional is in italic because tilt compensation was on and measurements' duration was managed by the controller, rather than the user.

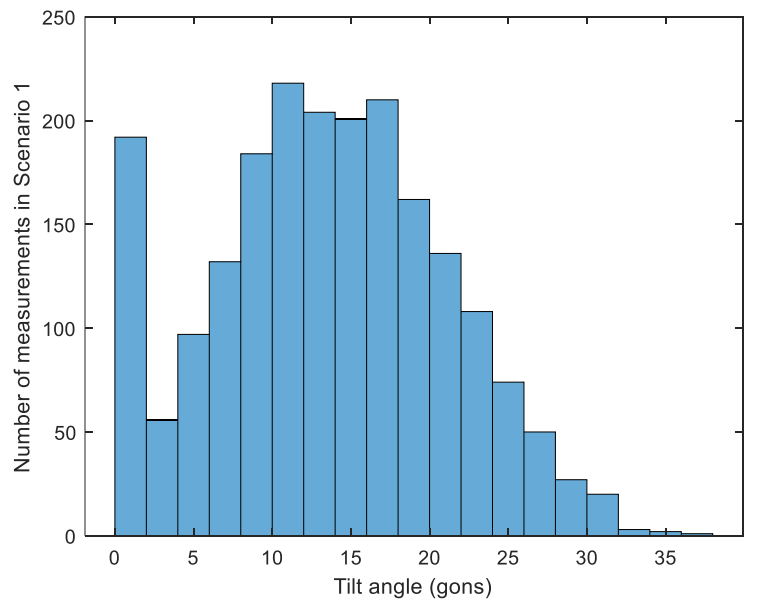

Figure 5. Histogram representing tilt angles of measurements acquired in Scenario 1

Figure 5 shows the histogram of the tilt angles (measured in gons) characterizing the measurements acquired in Scenario 1. It highlights there is a significant number of measurements in the range $[0,30]$. The first bin is more populated than others adjacent because the operators were requested, each time they visited a point, to acquire the first measurements keeping the pole vertical. Figure 6 shows the box plot of the 3D error for individual points and for the whole dataset. In order to save space, the $3 \mathrm{D}$ variable was only plotted. The box plot highlights that benchmarks 100 and 102 present more dispersed measurements and the latter shows an even severe behaviour; the other benchmarks show comparable results.

We were not surprised of what is highlighted by Figure 6, as point 100 has a wall just aside it (see Figure 2) and point 102 has a tree very close to it, whose crown is significantly protruding over the point.

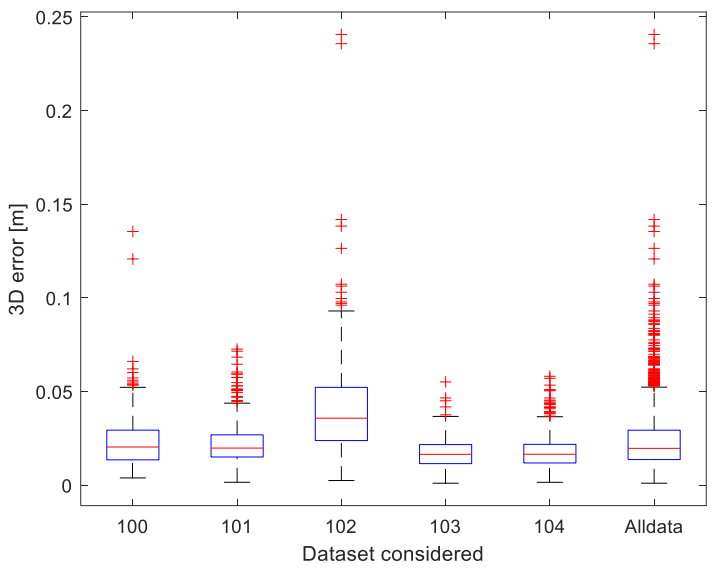

Figure 6. Box plot of the 3D error for individual points and for the whole dataset.

Figure 7 shows the histogram of the 3D error for single benchmarks and for the whole dataset. Outliers are particularly visible for the 102 point and are caused by occlusions, which are due to the presence of a tree. Outliers exist also for point 100, and here the cause is the presence of a high wall, which is very close to the point.

Histogram of the $3 D$ error
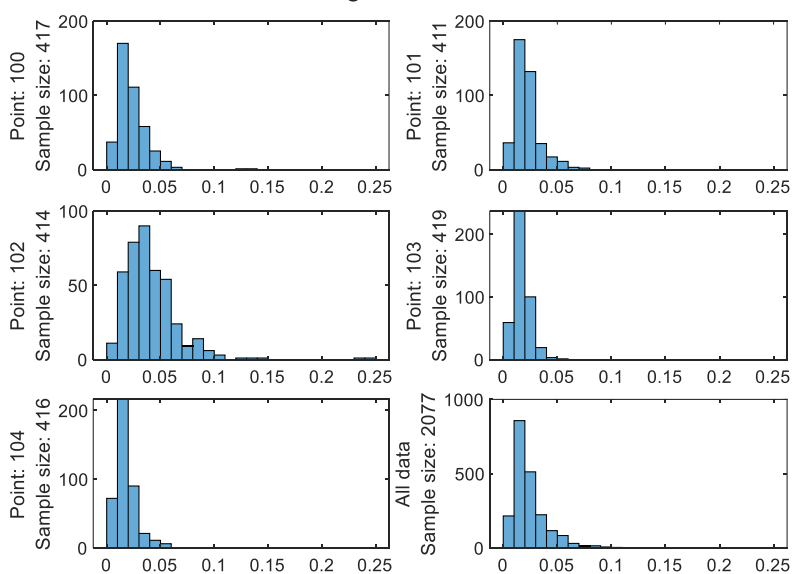

Figure 7. Histograms of $3 \mathrm{D}$ errors for the single points and for all the measurements together

The overall histogram suggests that almost all the measurements are within the $[0,0.06]$ range, for the $3 \mathrm{D}$ residual, thus showing a very good performance: indeed, the $95^{\text {th }}$ percentile has value $5.5 \mathrm{~cm}$. Nevertheless, outliers are clearly detectable, which we know the origin of, which is independent from the inherent behaviour of the used receiver. 
Therefore, data filtering was performed before extracting quality parameters. For each component, East, North and height, robust mean was estimated by the median operator. Robust standard deviation (std) was estimated based on the scaled MAD (Median Absolute Deviation): for normally distributed data $\left[x_{i}\right]$, it is known that

$$
\operatorname{std}\left(\left[x_{i}\right]\right)=1.4826 \mathrm{MAD}\left(\left[x_{i}\right]\right) 3 \sigma
$$

The robust $3 \sigma$ confidence interval was calculated for each component and one observation was considered an inlier if all its three components were inliers. By this way, 151 observations were discarded, out of 2077, corresponding to $7.27 \%$ of the total. Afterwards, descriptive statistics parameters were extracted for the whole datasets of the filtered measurements, without grouping them according to the point considered.

\begin{tabular}{|l|c|c|c|c|}
\hline & $\Delta \mathbf{E}[\mathbf{m}]$ & $\Delta \mathbf{N}[\mathbf{m}]$ & $\Delta \mathbf{h}[\mathbf{m}]$ & $\mathbf{d 3 D}[\mathbf{m}]$ \\
\hline $\min$ & -0.367 & -0.029 & -0.047 & 0.001 \\
\hline $\max$ & 0.024 & 0.028 & 0.057 & 0.061 \\
\hline mean & -0.006 & -0.001 & 0.005 & \\
\hline std & 0.010 & 0.010 & 0.017 & \\
\hline RMSE & 0.012 & 0.010 & 0.018 & \\
\hline Perc(68.27) & & & & 0.024 \\
\hline
\end{tabular}

Table 2. Descriptive statistics for the whole filtered dataset

Table 2 reports descriptive statistics for the single components and for the 3D error. The whole cleaned dataset is considered here, including all the point. The very usual parameters are shown, min, max, mean and std; we also report RMSE, that is the square root of the sum of squared mean with squared std. As std is the mean distance from the average value, RMSE is the mean distance from the true value, which is 0 . Indeed, residuals were calculated by forming the difference between NRTK-determined coordinated with the reference ones, which were measured with high precision and accuracy. RMSE values are around $1 \mathrm{~cm}$ for the planimetric components and of $1.8 \mathrm{~cm}$ for height.

For the 3D distance, some indicators are missing. We could have shown them, of course, but their interpretation is different, Indeed, single components are supposed to be normally distributed, while 3D distance clearly has another distribution, as the histograms reported in Figure 7 confirms. For single components, RMSE can be interpreted as the half-width of the interval having $68.27 \%$ probability; in analogy with that, for 3D error, we calculated the upper limit of the interval having $68.27 \%$ probability (the lower limit was set to 0); the corresponding value, which is comparable, to a certain extent, to RMSE, is 2.4 $\mathrm{cm}$ and highlights that the analysed measurements are highly precise and accurate. Other percentiles can be extracted, of course: the $95^{\text {th }}$ one has value $4.3 \mathrm{~cm}$, meaning that $95 \%$ of the inlying measurements have a $3 \mathrm{D}$ error lower or equal than the reported figure.

The dependence of uncertainty over the tilt angle was studied too. The set of the pole angles, represented in Figure 5, was subdivided into 10 unequal intervals having the same numerosity. Measurements were partitioned accordingly and the $68.27^{\text {th }}$ percentile was extracted for the 3D distance, for each bin. Figure 8 illustrates results and has, in abscissa, the mean tilt angle for each bin; in ordinate, the $68.27^{\text {th }}$ percentile is reported of $3 \mathrm{D}$ error. Also, the $95 \%$ confidence interval is reported, which was obtained with the bootstrap statistical method. The reported curve is not easily interpretable and does not confirm what was expected, i.e., the curve increases with the tilt angle. On the contrary, it decreases when angles are considered, in the range [5, 15] gons. For angles beyond 25 gons, a certain increase is visible indeed, but the RMSE figure is around $2.8 \mathrm{~cm}$, which is not too far from the overall average value of $2.4 \mathrm{~cm}$. It can also be observed that, in the range $[0,22]$ gons, empirical results are compatible with the hypothesis that 3D measurement error (at the 68.27 probability level) is lower than or equal to the overall average, $2.4 \mathrm{~cm}$. All in all, the rover seems very effective in compensating the pole's tilt.

\section{ACCURACY OF PHOTOGRAMMEYTRIC MEASUREMENTS}

Photogrammetric measurements of points shown in Figure 3 were also assessed. Preliminarily, coordinate conversion was performed, and a local cartesian reference system was adopted. The new $x$ axis is horizontal and parallel to the surveyed façade; it increases moving rightwards. The $y$ axis is vertical and parallel to the facade, too; in increases upwards. The $z$ axis is defined in order to form a right-handed coordinate system and increases when moving from the facade to the rover.

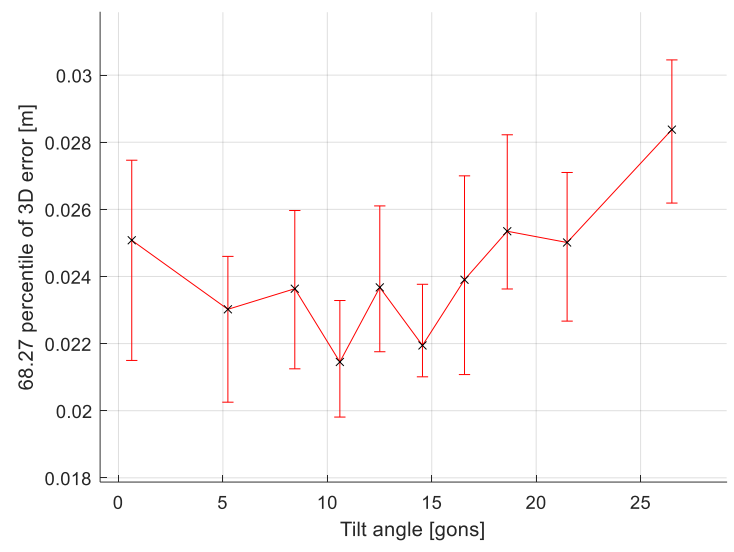

Figure 8. 3D error as a function of the tilt angle; more precisely, the $68.27^{\text {th }}$ percentile is shows, of $3 \mathrm{D}$ error, as explained in the text.

Residuals for the whole dataset
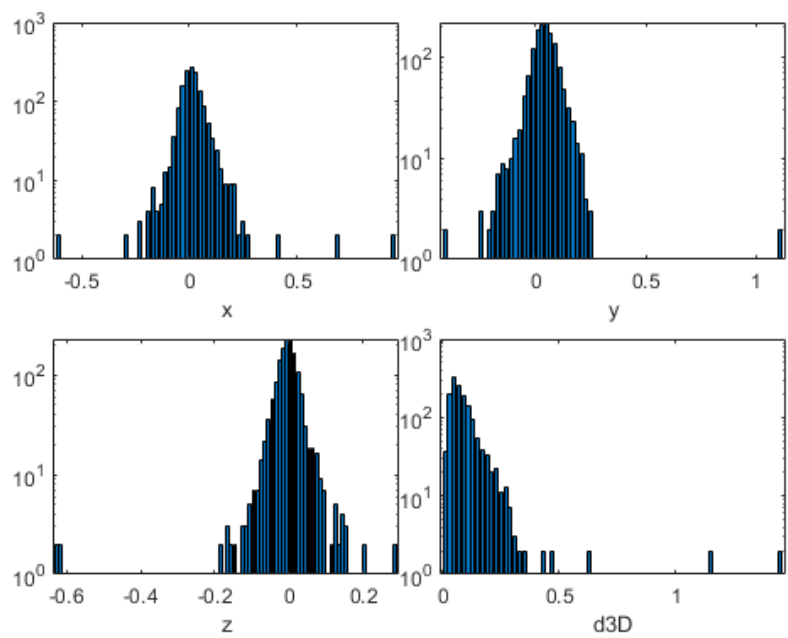

Figure 9. Histograms of the residuals of the whole dataset (all the users, all the points, all the distances); y scale is logarithmic, in order to make low-populated bins visible.

Figure 9 shows the histograms of the residuals for the whole dataset, including all the users, all the points and all the distances (4 to 12 meters). In order to make low-populated bins visible, logarithmic scale was set for the ordinate axis. Due to the 
properties of the logarithm function, the bin counts were incremented by 1 ; the lowest visible bins have a numerosity of 1 . As there are outliers, clearly, their filtering was performed, by applying the same methodology described in Section 8. Out of 1436 measurements, 137 were discarded, corresponding to $9.5 \%$ of the total. Pictures and results shown from here on, are related to the inliers only.

Exploratory analysis was preliminarily performed, of the residuals which were obtained by subtracting reference coordinated to the measured ones. Figure 10 reports the scatter plot for point 401: in the left-hand figure, the $x-y$ components are shown; in the right hand-figure, the $x-z$ plane is displayed.
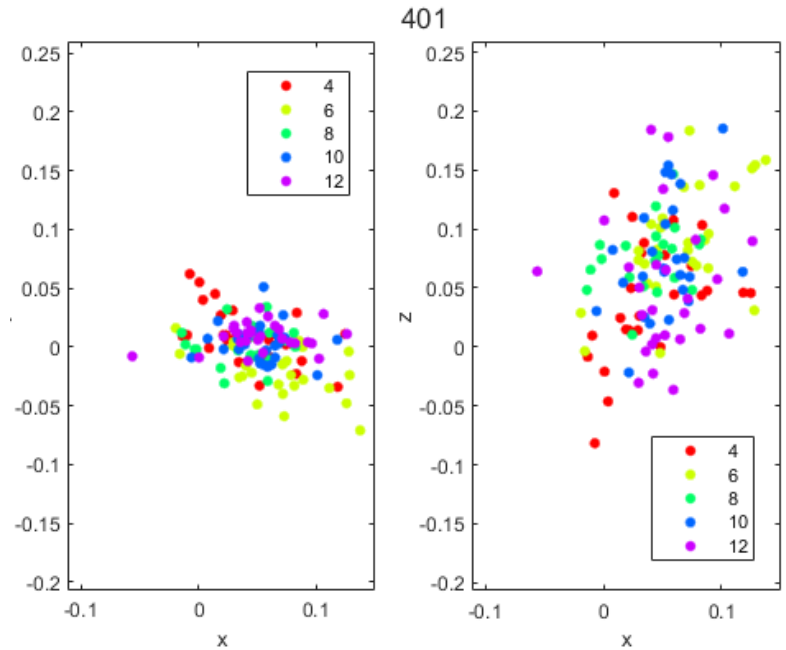

Figure 10. Scatter plot of the residuals for benchmark 401, for all the operators and all the considered rover-façade distance values.
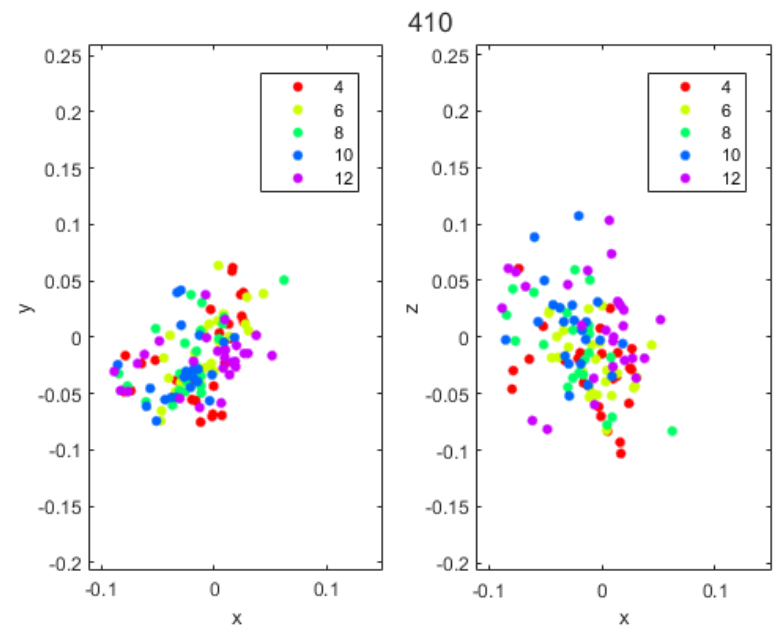

Figure 11. Scatter plot of the residuals for benchmark 410, for all the operators and all the considered rover-façade distance values.

Measurements acquired by all the operators and at all the distances are merged; nevertheless, dots are coloured according to the rover-facade distance (between 4 and 12 maters, with steps of 2), as the legend shows. We could ascertain that the performance of the three operators involved is comparable, while there is a moderate dependence on the distance, as it will be shown later in the paper.

Figure 10-left highlights that dispersion in $x$ is higher than in $\mathrm{y}$; Figure 10-right shows than dispersion in $z$ in greater than in $x$. This is a general behaviour, as confirmed by Figure 11, that is related to point 410 and by Figure 12 that refers to the whole dataset.

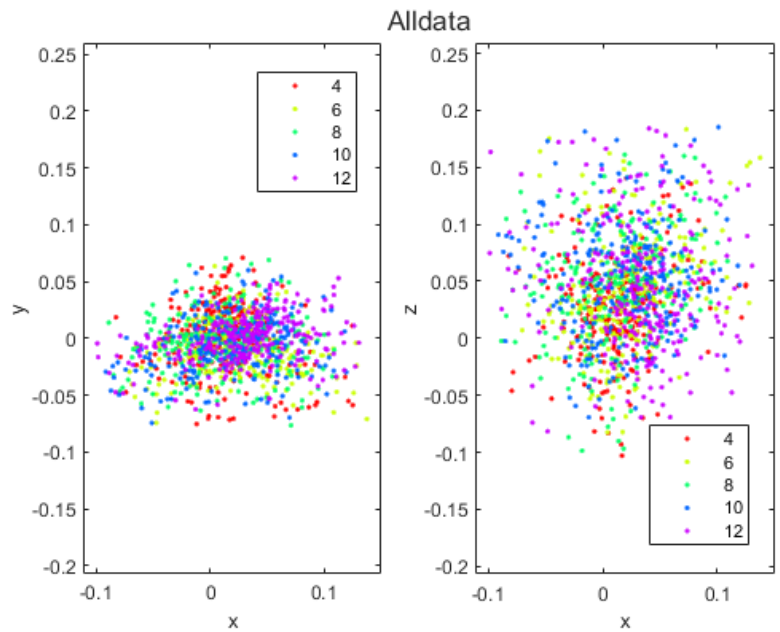

Figure 12. Scatter plot of the residuals for all the benchmarks, all the operators and all the considered rover-façade distance values.

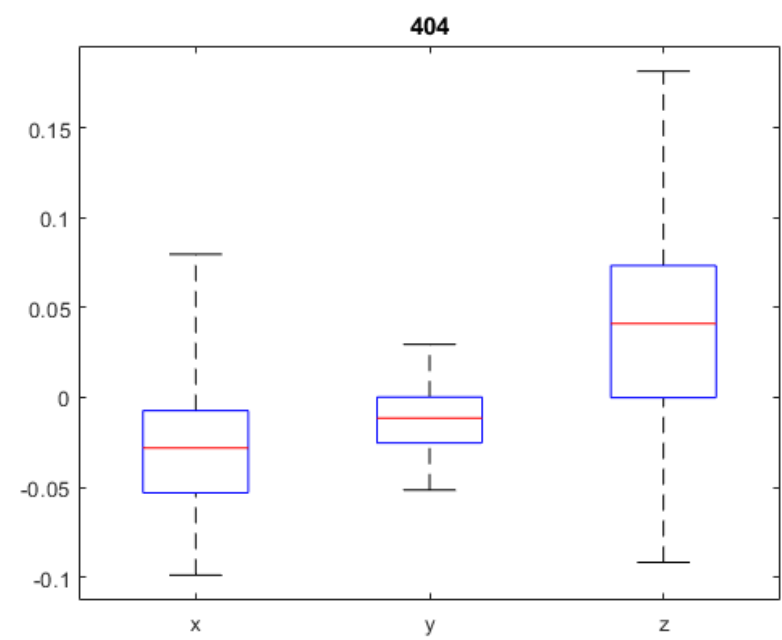

Figure 13. Boxplot for the $x, y$ and $z$ components of residuals, for the point 404 .

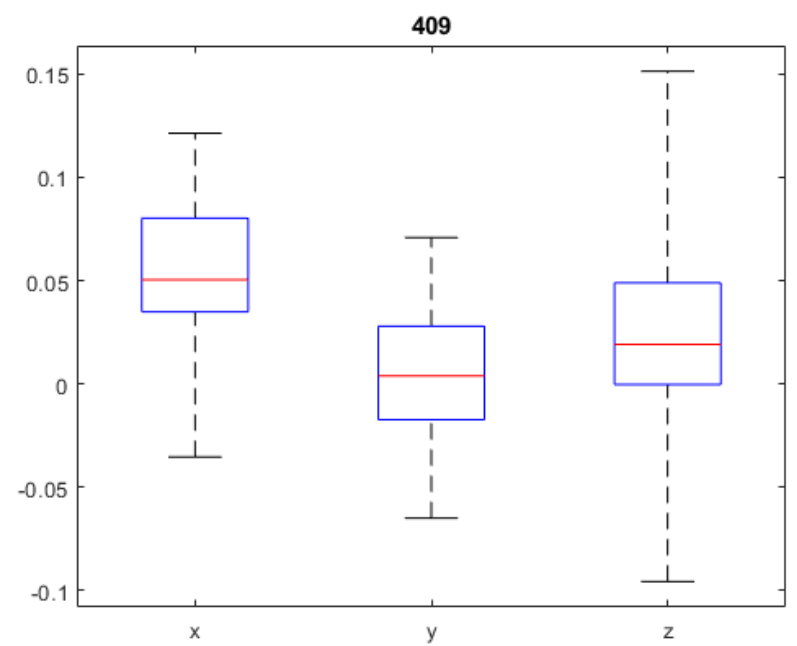

Figure 14. Boxplot for the $x, y$ and $z$ components and for the point 409 . 
Boxplots were also generated for all the points. In the following, a couple of examples will be presented only.

Figure 13 is for point 404, showing a behaviour that is comparable to the general one, in terms of measurements' dispersion, which is reported by the box's height. Indeed, $x$ is more dispersed than $y$ and $z$ is more than $x$.

There are exceptions, of course, and point 409 is such an example. Indeed, as Figure 14 illustrates, dispersion of the three components is approximately the same.

Descriptive statistics parameters were determined for all the inlying measurements, as reported by Table 3. As already reported, inliers 1299 out of 1436 measurements. Measurements are considered as inliers if all the three components lye inside the robust $3 \sigma$ robust confidence interval, where robust means that the central value is estimated by the median and the width is three times the scaled MAD (median absolute deviation); the scaled MAD is, in turn, the actual MAD times 1.4826 , that is, for normally distributed phenomena, the conversion factor between MAD and standard deviation.

Focusing on RMSE, being the average distance with respect to the reference value, estimated values are of 45,25 and $66 \mathrm{~mm}$, for the whole dataset, including all the points, the operators, and the configurations, in terms of the rover-facade distance. Systematics are also visible, which are limited in size. They are no further analysed in the present paper.

\begin{tabular}{|l|c|c|c|c|}
\hline & $\Delta \mathbf{E}[\mathbf{m}]$ & $\Delta \mathbf{N}[\mathbf{m}]$ & $\Delta \mathbf{h}[\mathbf{m}]$ & $\mathbf{d 3 D}[\mathbf{m}]$ \\
\hline ntot & 1436 & 1436 & 1436 & 1436 \\
\hline nin & 1299 & 1299 & 1299 & 1299 \\
\hline $\min$ & -0.010 & -0.077 & -0.103 & 0.004 \\
\hline max & 0.138 & 0.071 & 0.185 & 0.222 \\
\hline mean & 0.020 & -0.004 & 0.043 & \\
\hline std & 0.041 & 0.025 & 0.050 & \\
\hline RMSE & 0.045 & 0.025 & 0.066 & \\
\hline Perc(68.27) & & & & 0.086 \\
\hline
\end{tabular}

Table 3. Descriptive statistics for the whole dataset of the photogrammetric measurements.

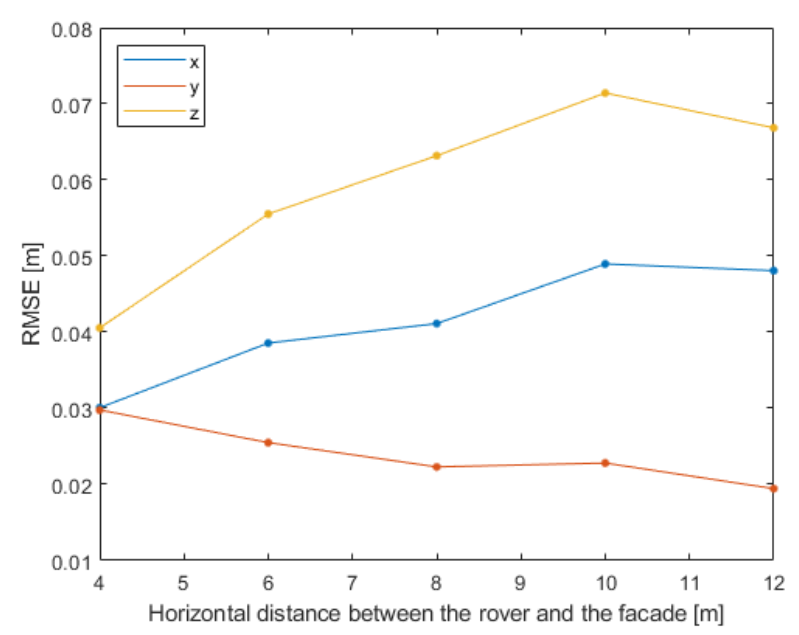

Figure 15. RMSE values for the $\mathrm{x}, \mathrm{y}$ and $\mathrm{z}$ components, as a function of the rover-faced distance.

One question could be raised, why overall accuracy indices have been extracted, when it is well known that quality of photogrammetric measurements linearly depend on the cameraobject distance. For sure we will investigate such aspect, in further papers. At the same time, we do not think that, in practical scenarios, users will be able to guarantee that the distance is constant. In order to take advantage of the GS18i rover, they must be free to measure any point being in the recommended range, from 4 to 10 metres.

As a last investigation, RMSE dependence on the camera-object distance was investigated. Measurements were grouped according to that parameter and quality parameters were extracted as before, for each of the available values, 4, 6, 8, 10 and 12 metres.

Figure 15 shows the determined curves and highlights some interesting phenomena, some counterintuitive. $\operatorname{RMSE}(\mathrm{x})$ and RMSE(z) increase with the distance, even if the curves flat down or decrease between 10 and 12 metres. On the other hand, RMSE(y) decreases with the distance and this is surprising.

\section{DISCUSSION, CONCLUSIONS AND FURTHER ACTIVITIES}

A first evaluation of the Leica GS18i rover has been performed. A test site has been created, at the University of Pavia, Italy. It includes 5 accessible points and 10 points belonging to a façade. Their reference coordinates have been determined with accurate and redundant surveying methodologies. The test site is highly demanding, due to the presence of multi-path sources and obstructions.

The accessible points have been determined by ordinary NRTK measurements, in which the pole was variously tilted. In total, 2077 measurements were acquired and processed. The rover proved to be highly capable to compensate for the tilt; overall, average accuracies are 12, 10 and $18 \mathrm{~mm}$, for the East, North and height components, in terms of RMSE values.

Points on the façade have been measurement by photogrammetry. A whole set of camera-object distances has been considered, ranging from 4 to 12 metres. Average RMSE values are of 45, 25 and $66 \mathrm{~mm}$, for $x, y$ and $z$ (with respect to a local reference system), including all the distances and all the points. In general, we think this is sufficient for the scope of visual positioning.

Undoubtedly, some aspects are difficult to explain, in terms of the general photogrammetric rules. Accuracy is significantly different for $\mathrm{x}$ and $y$. Moreover, $\operatorname{RMSE}(\mathrm{y})$ decreases when the distance increases, while the other two components behave as expected.

Such tricky aspects will be investigated by performing full photogrammetric processing of the acquired images. Indeed, the GS18i rover performs photogrammetry in a sort of black box style. It is said that the camera is calibrated, but the parameters of the model are not accessible; downloaded images are said to be undistorted and so they seem, but we could not check that so far. Also, it is said that the controller extracts tie points and performs bundle adjustment, but no information is given about this process. Finally, points were measured in the office using the standard way: the user clicks one feature on one image and the program matches it in several others. For the present paper, matches performed by the software supplied by the manufactures were not checked, but simply accepted: we can only assure that point identification performed by user was careful.

In next activities, full photogrammetric processing will be performed. Several other GCPs will be measured on the façade and the acquired photograms will be orientated by usual and full bundle adjustment. Moreover, if necessary, detailed simulation will be performed aiming at two main goals:

- $\quad$ to ascertain what is the actual error budget, i.e., what is the weight if the various error sources: direct measurement of exterior orientation, matching of homologous points, consistence and quality of tie points;

- to check whether the obtained measurement quality is the highest attainable, given the available instrumentation or 
can be improved via, e.g., refined camera calibration, improved tie points extraction, or different handling of the on-the-fly bundle adjustment.

\section{ACKNOWLEDGEMENTS}

The paper has been prepared within the frame of the CE4WE (Circular Economy for Water and Energy) research project, ID1139857, funded by the Lombardia Region, Italy.

Leica Geosystems Italia is acknowledged here (namely Eng. Filippo Quadranti and Eng. Davide Parmigiani) for lending us the rover we used for the first part of the test. Further activities, which are included in the present paper too, were performed with our sensor, after we bought it.

Three students at the University of Pavia performed the measurements, within the activities related to the preparation of their bachelor dissertation. They are Marco Raniolo, Davide Lodigiani and Alessandro Filippi. They proved to be enthusiast and highly committed.

Students' activity was carefully supervised by the authors and also by Paolo Marchese, a technician of the Laboratory of Geomatics, at the University of Pavia. $\mathrm{He}$ is gratefully acknowledged too.

\section{REFERENCES}

Arducam AR0134 [WWW Document], 2020. URL https://www.arducam.com/product/arducam-cmos-ar0134-1-3inch-1-2mp-color-camera-module-2/ (accessed 4.16.21).

Baiocchi, V., Piccaro, C., Allegra, M., Giammarresi, V., Vatore, F., 2018. Imaging rover technology: Characteristics, possibilities and possible improvements, in: Journal of Physics: Conference Series. Institute of Physics Publishing, p. 12008. https://doi.org/10.1088/1742-6596/1110/1/012008

Cera, V., Campi, M., 2017. Evaluating the potential of imaging rover for automatic point cloud generation, in: International Archives of the Photogrammetry, Remote Sensing and Spatial Information Sciences - ISPRS Archives. pp. 147-154. https://doi.org/10.5194/isprs-archives-XLII-2-W3-147-2017

El-Mowafy, A., 2000. Performance Analysis of the RTK Technique in an Urban Environment. Aust. Surv. 45, 47-54. https://doi.org/10.1080/00050353.2000.10558803

Feng, Y., Wang, J., 2008. GPS RTK Performance Characteristics and Analysis. J. Glob. Position. Syst. 7, 1-8. https://doi.org/10.5081/jgps.7.1.1

Leica GS18I [WWW Document], 2020. URL https://leicageosystems.com/products/gnss-systems/smart-antennas/leicags18i (accessed 2.5.21).

Luo, X., Schaufler, S., Branzanti, M., Chen, J., 2020. Assessing the benefits of Galileo to high-precision GNSS positioning RTK, PPP and post-processing. Adv. Sp. Res. https://doi.org/10.1016/j.asr.2020.08.022

Luo, X., Schaufler, S., Carrera, M., Celebi, I., 2018. HighPrecision RTK Positioning with Calibration-Free Tilt Compensation, in: FIG Congress.

Schaufler, S., 2020. GNSS Visual Positioning [WWW Document].
https://share.vidyard.com/watch/W5tQo7Dv1iGe5vmETzHTjR ? (accessed 4.19.21).

Schaufler, S., Fischell, M., Boffi, G., Luo, X., Rtk, G., 2020. An Innovative Image-Based Surveying Approach for Globally Referenced Remote Point Measurements An Innovative ImageBased Surveying Approach for Globally Referenced Remote Point Measurements ( 10354 ) Stefan Schaufler , Michele Fischell , Geo Boffi , Xiaog 10-14.

Takac, F., Zelzer, O., 2008. The relationship between network RTK solutions MAC, VRS ${ }^{\mathrm{TM}}$, PRS, FKP and i-MAX, in: 21st International Technical Meeting of the Satellite Division of the Institute of Navigation, ION GNSS 2008. pp. 2231-2238.

Visual Positioning and Leica GS18 I [WWW Document], 2020. URL https://leica-geosystems.com/products/gnsssystems/smart-antennas/leica-gs18i/of-visual-positioning-gs18-i (accessed 4.16.21). 